\title{
Evolution of the 2D surface structure of a silicon pitch grating under argon ion bombardment: experiment and modeling
}

Ivan Bizyukov $^{1,4}$, Andreas Mutzke ${ }^{2}$, Ralf Schneider ${ }^{3}$, James Davis ${ }^{4}$

${ }^{1}$ Karazin Kharkiv National University, Faculty of Physics and Technologies, 31 Kurchatov Ave., Kharkiv 61108, Ukraine

${ }^{2}$ Max-Planck-Institut für Plasmaphysik, EURATOM Association, Wendelsteinstr. 1, 17491

Greifswald, Germany

${ }^{3}$ Institute of Physics, Ernst-Moritz-Arndt University, Felix-Hausdorff-Str. 6, 17489 Greifswald, Germany

${ }^{4}$ University of Toronto Institute for Aerospace Studies, 4925 Dufferin Street, Toronto, Ontario, Canada M3H 5 T6

\begin{abstract}
This work aims at validation of the newly developed SDTrimSP-2D code by comparing experiment measurements and modeling of the evolution of a 2D surface structure under argon ion bombardment. SDTrimSP-2D allows for the simulation of ion-surface interactions with 2D micro-structured surfaces, where the first dimension is the depth and the second is parallel to the surface. The main advantage of the approach implemented in SDTrimSP-2D code is that the interdependency of surface morphology and sputtering is naturally taken into account. The code follows the evolution of surface morphology and provides a detailed nanoscale description of processes characterizing the ion-surface interactions. In this work, a Si pitch grating with typical dimensions of 200-250 nm, provides a well-characterized 2D system. This is bombarded with 6 $\mathrm{keV} \mathrm{Ar}^{+}$ions at normal incidence angle as well as at an angle of $42^{\circ}$ both parallel and perpendicular to the pitch grating structure. Cross-sections of the bombarded Si pitch grating were obtained by SEM and compared to the calculated surface profile revealing good agreement between experiment and simulation. The calculations also provide improved insight into the mechanisms of grating erosion and material transport by redeposition.
\end{abstract}

Keywords: SDTrimSP-2D, sputtering, redeposition, surface morphology, local ion-surface interactions.

PACs numbers: [reserved for the case if needed] 


\section{Introduction}

Sputtering of a surface by bombardment with energetic ions is a physical process which plays an important role in many plasma devices ranging from small magnetrons to large tokamaks. Extensive experimental and theoretical research on ion-surface interactions has been conducted in the past [1] leading to a general understanding of the basic physics governing the interaction of ions with surfaces. This has been confirmed by the very good agreement of sputter yield values for many ion-target combinations as measured in experiments and as calculated by numerical models [2].

However, existing codes have only limited ability to simulate the sputtering of surfaces with developed morphology or surfaces with inherent roughness. Numerical simulation of the interaction of ions with a non-planar surface can lead to new perspectives for computational prediction of surface nano-structure formed during ion implantation or deposition. During the past decade, there have been a number of approaches for the numerical simulation of ion-surface interactions for surfaces including surface morphology effects. Ruzic [3] suggested the implementation of surface roughness as a fractal geometry. Another approach, developed by Kuestner et.al., focused on predicting the increase of sputter yields through representing the surface by aggregates of simple surfaces at tilted angles [4][5]. Although reasonable agreement with experiment has been achieved, both approaches are limited because the surface morphology is not considered to be influenced by sputtering and (re)deposition.

The SDTrimSP-2D code [6], which shares the physical model implemented in codes of the TRIM family, is an extension of previous codes and is capable of simulating the bombardment of 2D micro-structured surfaces. A typical example of such a 2D system is a diffraction lattice. Although the model has already shown its ability to predict the modification of rough surfaces qualitatively [7][8], it still requires validation by ion beam experiments with well-characterized surface morphology.

In this work, a silicon pitch grating has been irradiated by a $6 \mathrm{keV} \mathrm{Ar}^{+}$ion beam at normal incidence angle as well as at angles of $42^{\circ}$ both parallel and perpendicular to the structure (Figure 1). These three bombardment cases modify the surface morphology of the pitch grating in significantly different ways, allowing a detailed benchmarking of the code. SDTrimSP-2D simulations of modified surface profiles are compared to scanning electron microscope (SEM) 
images following the bombardment. The calculations reveal nanoscale effects governing the modification of surface morphology

\section{Experimental procedures}

Figure 2 shows a typical SEM image of the pitch grating cross-section in an area outside of the ion beam. The nano-structured specimen is fabricated on a $\mathrm{Si}$ wafer with an intermediate $\mathrm{Ta}$ layer with a thickness of $650 \mathrm{~nm}$. The Ta layer is used as a reference marker to allow quantitative measurements of the Si layer thickness. The period of the structure has been designed to be $500 \mathrm{~nm}$ ( $250 \mathrm{~nm}$ for pits and $250 \mathrm{~nm}$ for grates); the height of the grates is $200 \mathrm{~nm}$. The actual grating dimensions deviate from the nominal values by $5-20 \mathrm{~nm}$, which imposes a lower limit on the agreement between the experimental results and calculations. Although the code can accept any surface profile as initial condition, the nominal structure parameters were used because they are considered to be a representative average. Typical differences between the specimen crosssection and the initial surface profile used in simulations is shown in (a).

The pitch grating specimens were irradiated in the UTIAS dual-beam mass-separated ion accelerator [9] with a beam of $6 \mathrm{keV} \mathrm{Ar}{ }^{+}$ions. The angle of incidence was fixed to be either perpendicular to the macroscopic plane of the specimen, or at an angle of $42^{\circ}$. The bombarded area was partly covered with aluminum foil to provide an untouched surface adjacent to the irradiated one, providing a sharp transition from the virgin surface to the eroded one.

The experimental fluence was derived from a measurement of the ion beam current and the beam spot area. The ion beam current was $5 \mu \mathrm{A}$ over a beam spot with typical diameter of 4$5 \mathrm{~mm}$, such that the average flux was $\approx 3 \times 10^{18} \mathrm{~m}^{-2} \mathrm{~s}^{-1}$. For the experiment at normal incidence, the fluence was $50-80 \times 10^{20} \mathrm{~m}^{-2}$; at tilted angles the fluence was $10-20 \times 10^{20} \mathrm{~m}^{-2}$. Since the beam spatial distribution is expected to be approximately Gaussian, strong variations in flux and fluence are expected across the exposed area, and particularly towards the periphery of the exposed region.

Following the bombardment, the specimens were extracted from the vacuum system and cracked to achieve the cross-sectional view. The cross-section was studied using a highresolution Hitachi S-5200 Scanning Electron Microscope.

A series of cross-sections following the ion exposure, together with the simulated surface profiles, are shown in . These show the evolution of the surface morphology under ion 
bombardment. Note that the SEM images were taken outside the region immediately adjacent to the $\mathrm{Al}$ foil to avoid the possibility of redeposited $\mathrm{Al}$ atoms contributing to the evolution of the surface morphology. The variations observed in the surface modification are a result of the nonuniform (Gaussian) beam profile, such that the ion current density is gradually reduced towards the edges of the irradiated spot. As a result, the central part of the bombarded surface is irradiated with a higher fluence, while edges are less exposed. Thus, fracturing the specimens along the diameter of the beam spot produced a cross-section of the surface with varying ion beam exposure. Using SEM, a series of cross-section images were taken at different locations (i.e. at different radii of the spot) experiencing different fluences.

\section{The SDTrimSP-2D program}

Essentially, SDTrimSP-2D is a 2-D extension of SDTrimSP [10], which, in turn, is a generalized version of the TRIDYN program [11]. It can be run in static or dynamic mode (SD) on sequential or parallel systems (SP). SDTrimSP-2D uses a 2-D mesh to represent the surface morphology, the first dimension is the direction perpendicular to the macroscopic surface plane, and the second is in a direction parallel to that plane. This representation is sufficient to simulate the ion bombardment of surfaces with $2 \mathrm{D}$ micro-structure extended into the $3^{\text {rd }}$ dimension. It shares the same physical model of ion-surface interactions with other codes of the TRIM family. However, the resolution of a second dimension requires a 2-D domain with separate cells.

The code follows the density changes in the target material due to projectile and recoil particles coming to rest after a complete slowing-down at the end of their trajectories. In SDTrimSP/TRIDYN, this is done by a 1-D relaxation of the cells. Each trajectory creates a mass flux in the cells it passes. These fluxes can act as sink or source terms for the particle densities. To ensure particle conservation within the numerical setup, which uses a 1D grid of cells in which each cell has a constant volume density according to the material, volume changes of the 1D cells (expansion or contraction perpendicular to the surface) are used to represent changes to the number of particles in a cell. In SDTrimSP-2D, this procedure has been extended to 2D, subject to the requirement that all volume changes applied are divergence free. This reflects particle conservation in the projectile-target system expressed by volume changes. For each cell, the resulting mass fluxes (representing the transfer of particles into or out of the cell) are taken to be anisotropic by introducing the anisotropy coefficient $\left(K_{\text {anis }}\right)$ of the volume relaxation. This 
anisotropy coefficient defines the ratio of horizontal volume changes (representing horizontal mass fluxes parallel to the surface) and perpendicular volume changes (representing mass fluxes in vertical direction). The horizontal transport (parallel to the surface) is usually set smaller than the vertical one, because swelling or shrinking are primarily observed experimentally in the vertical direction. In the simulations presented here an anisotropy coefficient of 0.5 was used. Thus, the cells at the surface exposed to incident ions can change in two directions. The volume of cells without sides bordering on the surface is kept fixed. The relaxation process is done in several iterations until the divergence of the mass fluxes (transfer of particles between cells) become zero and steady-state conditions without internal tension are obtained. From this steadystate, divergence-free solution the volume changes are applied. In addition, splitting and annihilation of cells was introduced in SDTrimSP-2D, according to a maximum and minimum number of atoms, to be able to represent creation of holes or strong deposition.

The program also includes outgassing, which is responsible for the transport of noble gas atoms towards the surface after their slowing-down in the target. The outgassing flux, $J$, through a surface with a transport coefficient, $K$, is :

$$
J=-K \times c
$$

and the corresponding fluence dependence of the concentration $c$ :

$$
\frac{\partial c}{\partial f}=-K \frac{\partial c}{\partial x}
$$

where $f$ is the local fluence and $x$ is the distance to the surface. At each fluence step, a certain amount of gas atoms is moved towards the surface. The amount depends on the concentration, $c$, of gas atoms (atoms per volume) in the layer and the outgassing coefficient $K$. This coefficient was determined by comparison with experimental data; for Ar in Si the outgassing coefficient is $K=65 \times 10^{-24} \mathrm{~cm}^{3} /$ ion [12].

Since the surface is periodic structure in the lateral direction, periodic boundary conditions in this direction are used.

Additional diagnostics are used to analyze the results of the numerical computations and are able to provide local values of the sputtering and redeposition yields. The local sputtering yield calculates, how many Si atoms have been removed from a given cell normalized to the number of incident Ar atoms arriving in this cell. For a given surface cell, it is calculated as

$$
Y=N_{\text {sputter }} / N_{\text {incident }}
$$


where $N_{\text {sputter }}$ is the number of Si atoms removed from the cell, and $N_{\text {incident }}$ is the number of incident Ar ions. Note that Si atoms lost from one cell may end up in another or may pass through several cells before reaching the surface. Similarly, incident $\mathrm{Ar}^{+}$ions may have first passed through other cells.

To keep track of these events, we use three different sputter yields: $Y_{\text {local }}, Y_{\text {removed }}$ and $Y_{\text {static }}$. For the calculation of $Y_{\text {local }}$, the number of particles counted as sputtered Si atoms is measured at some distance away from the surface to exclude those particles which are redeposited. For $Y_{\text {removed }}$ all particles removed from the surface are taken into account, independent of their subsequent paths; thus redeposited particles are also included. $Y_{\text {static }}$ is calculated by SDTrimSP and it represents the sputter yield of a planar Si surface bombarded by Ar ions at the local angle of incidence.

Similar to the sputtering yield, the redeposition "yield" calculates the number of all previously sputtered $\mathrm{Si}$ atoms passing through the surfaces of a cell, again normalized to the number of incident Ar atoms in this cell. It is calculated as:

$$
\operatorname{Re}_{\text {local }}=N_{\text {implanted }} / N_{\text {incident }}
$$

where $N_{\text {implanted }}$ is the number of previously sputtered Si atoms, which were deposited in a particular cell; $N_{\text {incident }}$ is the number of incident Ar ions in this cell. Both values refer to all particles travelling through a given local cell at the surface. Atoms may ultimately be deposited deeper in the material, however, the deposition event is counted in the local redoposition yield only for the cell through which it has penetrated into the specimen All incident Ar atoms are also accounted for in the same way.

The introduction of the two yields, $Y_{\text {local }}$ and $Y_{\text {removed }}$ is useful in the analysis of the particle transport effects. The source of redeposited particles are the surfaces at which $Y_{\text {removed }}-Y_{\text {local }}>0$, and the larger the difference between these two yields the stronger the contribution to the redeposition. The particles are transported to and redeposited on surfaces with $\operatorname{Re}_{\text {local }}>0$; net deposition occurs on surfaces for which $R e_{\text {local }}>Y_{\text {removed }}$.

\section{Results and discussion}

\subsection{Bombardment at normal angle of incidence}

The comparison of experiment and simulation is shown in for a series of fluences. The simulated surface profiles are overlaid in red on the SEM photos. In the vertical planes of Figure 
3 , the bottom of the square has been matched to the interface between the Ta layer and the $\mathrm{Si}$ grating; in the horizontal plane the vertical axes of symmetry were aligned.

With increasing ion fluence, the rectangular surface structure is first rounded, and then becomes pointed. As indicated by the location of the Ta interlayer, the pitch grating is sputtered at both the raised features and the valleys. Over the range of simulated fluences, the calculations provide an accurate progression of the surface structure, to within the initial accuracy of the pitch grating structure, $5-20 \mathrm{~nm}$. While the experimental ion fluences cannot be determined at individual points within the beam spot area, the calculated fluences $\left(10-60 \times 10^{20} \mathrm{~m}^{-2}\right)$ are consistent with those of the experiment, $50-80 \times 10^{20} \mathrm{~m}^{-2}$ in the central region of the beam spot and lower towards the edges. This consistency provides an independent validation of the sputtering yields calculated by the model.

Thus, the model exhibits excellent agreement between the experimental and calculated surface profiles. It can further be used to reveal details of the dynamics of ion-surface interactions (especially sputtering and redeposition), which are not accessible experimentally. shows a series of the calculated surface profiles and the respective sputtering and redeposition yields, as well as local angles of incidence for given fluence values. Comparing them, one can see the relationship between macro- and nanoscale dynamics of the ion-surface interactions. In general, surfaces with higher angle of incidence are sputtered most intensively, as indicated by $Y_{\text {removed }}$ and, therefore, give the highest contribution to the total sputtering yield. At all fluences, the difference between $Y_{\text {removed }}$ and $Y_{\text {local }}$ is rather pronounced on tilted surfaces; about half of the sputtered atoms are redeposited. This occurs due to a combination of forward sputtering and the relatively large height of the structure intercepting the sputtered particles. At fluences larger than $20 \times 10^{20} \mathrm{~m}^{-2}$ the difference between sputtering and redeposition increases with fluence. The reason being that the structure height becomes smaller, which is not favorable for redeposition. Therefore, the number of escaping particles increases at the expense of the number of redeposited particles.

Calculations show that the overall yield peaks at a fluence of $20 \times 10^{20} \mathrm{~m}^{-2}$. At this point, the structure exposed to the ion flux is characterized by the largest number of surfaces with high slopes relative to the incident ion direction. For lower fluence, the number of such surfaces was lower; for larger fluence the slope of the sputtered surfaces was decreased. The local sputtering and redeposition yields become constant across the structure at fluences larger than $70 \times 10^{20} \mathrm{~m}^{-2}$, 
as the surface morphology has become almost completely flat. At the same time, total yield becomes constant with fluence.

It is worth noting, however, that the redeposition yield is still above zero at high fluences, which is explained by nano-roughness of the simulated surface, which has a typical value is 1$3 \mathrm{~nm}$. At the moment it is not possible to distinguish, whether this is an artifact of the numerical model or a real physical effect.

\subsection{Bombardment at an angle of $42^{\circ}$ parallel to the structure}

One might speculate that if the incidence angle is changed in the plane parallel to the structure, the surface morphology evolution would be similar to the situation at normal incidence only with higher sputter yields. However, the experiment shows a significantly different behavior: the development of triangle-shaped structures as shown in . The matching of the simulated profiles to the experimental ones in the plots has been performed as described in the previous subsection.

Estimation of the experimental fluence provides an average value of $10-20 \times 10^{20} \mathrm{~m}^{-2}$, which is somewhat lower than that required by the simulation $\left(5-40 \times 10^{20} \mathrm{~m}^{-2}\right)$. However, $40 \times 10^{20} \mathrm{~m}^{-2}$ would not be an unreasonable estimation of the maximum experimental fluence at the centre of the beam spot. Estimation of the experimental fluence provides an average value of $10-20 \times 10^{20}$ $\mathrm{m}^{-2}$, with peak values perhaps 2-3 times larger. Again, this is consistent with that required by the simulation $\left(5-40 \times 10^{20} \mathrm{~m}^{-2}\right)$.

Again, the code is able to follow the evolution of the surface profile with an accuracy of 5$20 \mathrm{~nm}$. Although the code has been developed to work with 2D micro structured surfaces, the trajectories of projectiles and recoils are all calculated in 3D, giving a correct simulation of sputtering and redeposition. While the surface structure initially has a rectangular shape, by a fluences of $30 \times 10^{20} \mathrm{~m}^{-2}$ it has become triangular. With further bombardment, the triangle-shaped structure is retained, but the size of the triangles decreases until the surface profile becomes similar to that shown in (h). Beyond a fluence of $40 \times 10^{20} \mathrm{~m}^{-2}$, the pitch grating has been eroded down to the Ta interlayer, thus making further comparisons with calculations impossible.

Sputtering and redeposition yields together with local angles of incidence are shown in . At a fluence of $10 \times 10^{20} \mathrm{~m}^{-2} \mathrm{Si}$ atoms are primarily sputtered from the upperparts of the structure, while the redeposition occurs more towards the lower parts. The difference in the location of the 
dominant sputtering and redeposition processes indicates a significant particle transfer from one location to another. Thus it appears that the triangle-shaped structure is formed and maintained by the redeposition of $\mathrm{Si}$ atoms from the top of the structure down to its base.

Up to a fluence of $20 \times 10^{20} \mathrm{~m}^{-2}$ the transfer of Si atoms appears similar to that at normal incidence. however, in the case of normal incidence, redeposition is almost everywhere compensated by sputtering, while at the larger angle of incidence, deposition still contributes to the growth of the triangle base. At the inclined angle of incidence, the local angle of incidence always stays above $42^{\circ}$, resulting in higher sputtering yield of Si atoms, and consequently greater deposition nearby.

\subsection{Bombardment at an angle of $42^{\circ}$ perpendicular to the structure}

While the previous two cases have provided examples of symmetrical spatial distributions of sputtering and redeposition along the structure, bombardment at an inclined angle perpendicular to the structure will produce strongly non-symmetrical profiles. In this case, the structure is modified to a scalene triangle. As seen in the code is again able to follow the evolution of the profile with the same accuracy of 5-20 nm as found for the previous two cases. As in Section 4.2, the experimental and calculated fluences are fully consistent.

The spatial distribution of the sputtering and redeposition yields in relation to the actual profile is shown in . Initially, redeposition is provided only by Si atoms removed from the right face of the feature (located near position $+100 \mathrm{~nm}$ in at a fluence of $5 \times 10^{20} \mathrm{~m}^{-2}$ ), because the rest of the exposed surface is located on the top of the structure and $\mathrm{Si}$ atoms removed from there cannot reach the lower parts of the structure. Also, the redeposition yield is extremely low at the top as long as it keeps its relatively flat shape (to a fluence of $\sim 10 \times 10^{20} \mathrm{~m}^{-2}$ ).

At fluences larger than $10 \times 10^{20} \mathrm{~m}^{-2}$ there are two locations where the redeposition yield peaks. One of them is located on the initially vertical surface (left face) in the region between -100 and $-200 \mathrm{~nm}$, which is shadowed and, therefore, not eroded. Instead, sputtered Si atoms are mainly absorbed by this surface due to its vertical arrangement favorable to intercepting particles. A smaller fraction of the Si atoms are deposited in the initial horizontal region located between +150 and $+250 \mathrm{~nm}$, which is also shadowed. As the fluence is increased, these regions are joined together accompanied by the disappearance of the shadowed region. When the shadowed region disappears completely, the structure profile takes the shape of a scalene triangle 
with the angle determined by the beam incidence (see, fluence $25 \times 10^{20} \mathrm{~m}^{-2}$ ). The left-hand side of the triangle is the region of greatest deposition, however, it is effectively sputtered by the near-glancing beam incidence. In contrast, the sputtering and deposition are approximately equal on the right side of the triangle, which is now exposed to the ion flux at (approximately) normal angle.

The simulation of this system for higher fluence show that the triangular shape is more and more smoothened and then tends to get flat.

\section{Conclusions}

The Interaction of a $6 \mathrm{keV} \mathrm{Ar}{ }^{+}$ion flux with a Si pitch grating structure was studied experimentally and by means of numerical simulation using the SDTrimSP-2D code. The pitch grating were bombarded at normal incidence as well as at an angle of $42^{\circ}$, both parallel and perpendicular to the structure. The evolution of the surface morphology (i.e. cross-sections of the specimens), observed in experiment by SEM, has been compared directly to the results of the simulation. In all three cases, this comparison has shown agreement between the experimental and simulated profiles to be within the typical experimental initial deviation of 5-20 nm. The simulated results allowed a detailed description of the nanoscale processes accompanying the erosion: local sputtering, redeposition, and transport of the sputtered Si atoms from one surface to another.

In general, the SDTrimSP-2D code has been shown to provide a valid simulation model for the interaction of recycling ion fluxes with 2D surfaces. The comparison still contains some uncertainty with regard to the absolute sputtering yields, as the spatial variations in ion fluence can only be estimated from the measurements of beam current and spot size. However, estimation of the variations in fluence over the beam spot are consistent with the values required by the calculations, providing a reasonable independent verification. This question will be addressed in future work, where the fluence dependent sputtering yields will be compared to those calculated by the SDTrimSP-2D code. In addition, the capabilities of the code are limited to collisional cascade effects and do not include chemical effects, radiation-enhanced sublimation, etc., which (at least qualitatively) will be included later. 


\section{Acknowledgements}

Authors would like to thank Dr. I. Gourevich from Center for Nanostructure Imaging, Department of Chemistry, University of Toronto. Authors would like to thank Dr. K. Krieger, Materials Research Division, IPP Garching, for helpful discussions in sample design and providing the samples used in this study. Financial support for the experimental work performed at the University of Toronto was provided by the Natural Sciences and Engineering Research Council of Canada through a grant to Prof. A. A. Haasz. 


\section{References}

1 R. Behrisch (ed). Sputtering by Particle Bombardment. I. Physical Sputtering of SingleElement Solid. ISBN 978-3-540-10521-3 (Berlin, Springer, 1979).

2 R. Behrisch, W. Eckstein (ed). Sputtering by Particle Bombardment. Experiments and Computer Calculations from Threshold to MeV Energies. ISBN 978-3-540-44500-5 (Berlin, Springer, 2007).

3 D. Ruzic. The effects of surface roughness characterized by fractal geometry on sputtering. Nucl. Instrum. Meth. vol.B47, pp. 118-125, (1990).

4 M. Kuestner, W. Eckstein, V. Dose, and J. Roth. The influence of surface roughness on the angular dependence of the sputter yield. Nucl. Instrum. Meth. vol.B145, pp. 320-331, (1998).

5 M. Kuestner, W. Eckstein, E. Hechtl, and J. Roth. Angular dependence of the sputtering yield of rough beryllium surfaces. J. Nucl. Mater. vol.265, pp. 22-27, (1999).

6 A. Mutzke, R. Schneider. SDTrimSP-2D: Simulation of Particles Bombarding on a Two Dimensional Target Version 1.0. IPP Report 12/4 (Garching, Max-Planck-Institute for Plasmaphysics, 2009).

7 A. Mutzke, R. Schneider, and I. Bizyukov. SDTrimSP-2D studies of the influence of mutual flux arrangement on erosion and deposition. J. Nucl. Mater. vol.390-391, pp. 115-118, (2009). 8 I. Bizyukov, A. Mutzke, R. Schneider, A. Gigler, and K. Krieger. Morphology and changes of elemental surface composition of tungsten bombarded with carbon ions. Nucl. Instrum. Meth. vol.B266, pp. 1979-1986, (2008).

9 J. W. Davis, A. A. Haasz. Synergistic chemical erosion of graphite due to simultaneous bombardment by $H+$ and other low-Z ions using a dual-beam accelerator. Nucl. Instrum. Meth. vol.B83, pp. 117-124, (1993).

10 W. Eckstein, R. Dohmen, A. Mutzke, and R. Schneider. SDTrimSP: A Monte-Carlo Code for Calculating Collision Phenomena in Randomized Targets. IPP Report 12/3 (Garching, MaxPlanck-Institute for Plasmaphysics, 2007).

11 W. Moeller, W. Eckstein, and J. P. Biersack. TRIDYN - binary collision simulation of atomic collisions and dynamic composition changes in solids. Comput. Phys. Commun. vol.51, pp. 355368, (1988).

12 A. Mutzke, W. Eckstein. Ion fluence dependence of the Si sputtering yield by noble gas ion bombardment. Nucl. Instrum. Meth. vol.B266, pp. 872-876, (2008). 


\section{List of Figure Captions}

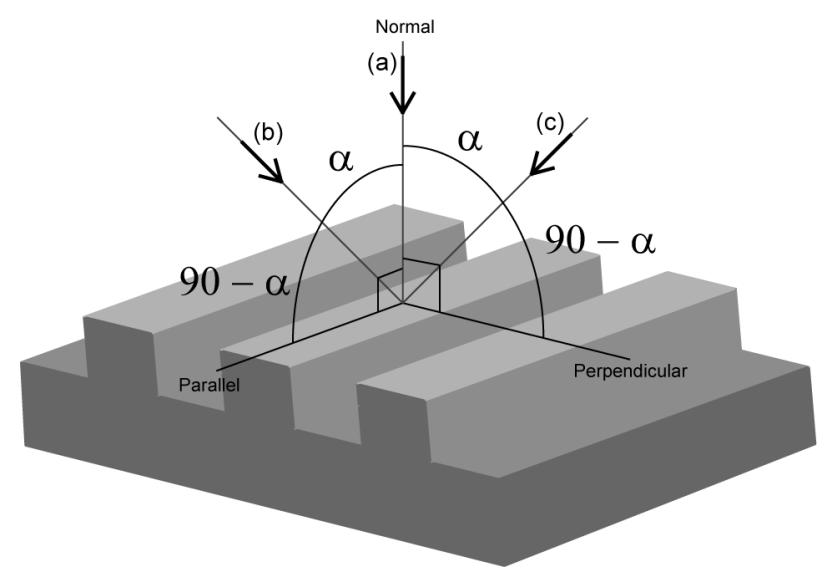

Figure 1. The direction of the incident ion beam on the pitch grating: (a) - normal angle of incidence, (b) - incidence at an angle of $42^{\circ}$ parallel to the structure and (c) - incidence at an angle of $42^{\circ}$ perpendicular to the structure.

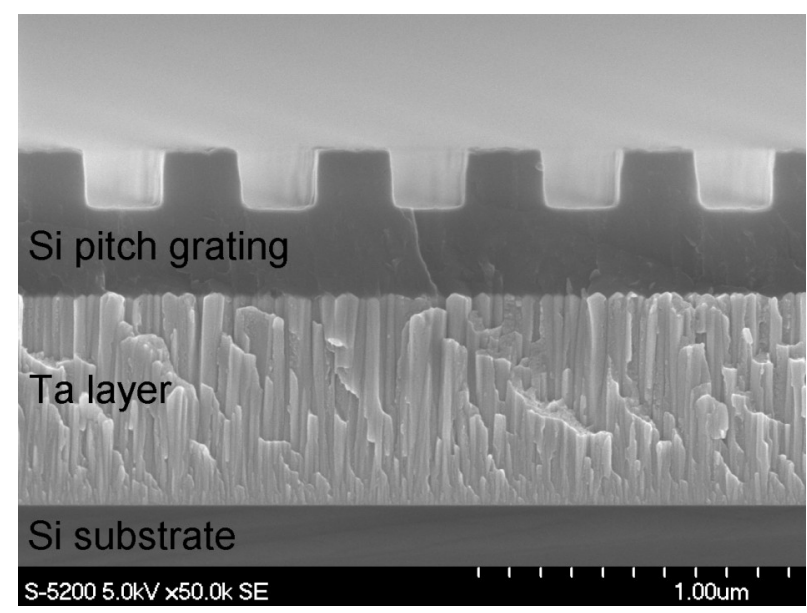

Figure 2. SEM image of a cross-section of the Si pitch grating. Silicon wafer (the substrate) is covered by $650 \mathrm{~nm}$ Ta layer. On top of it Si pitch grating structure manufactured by means of lithography methods. 

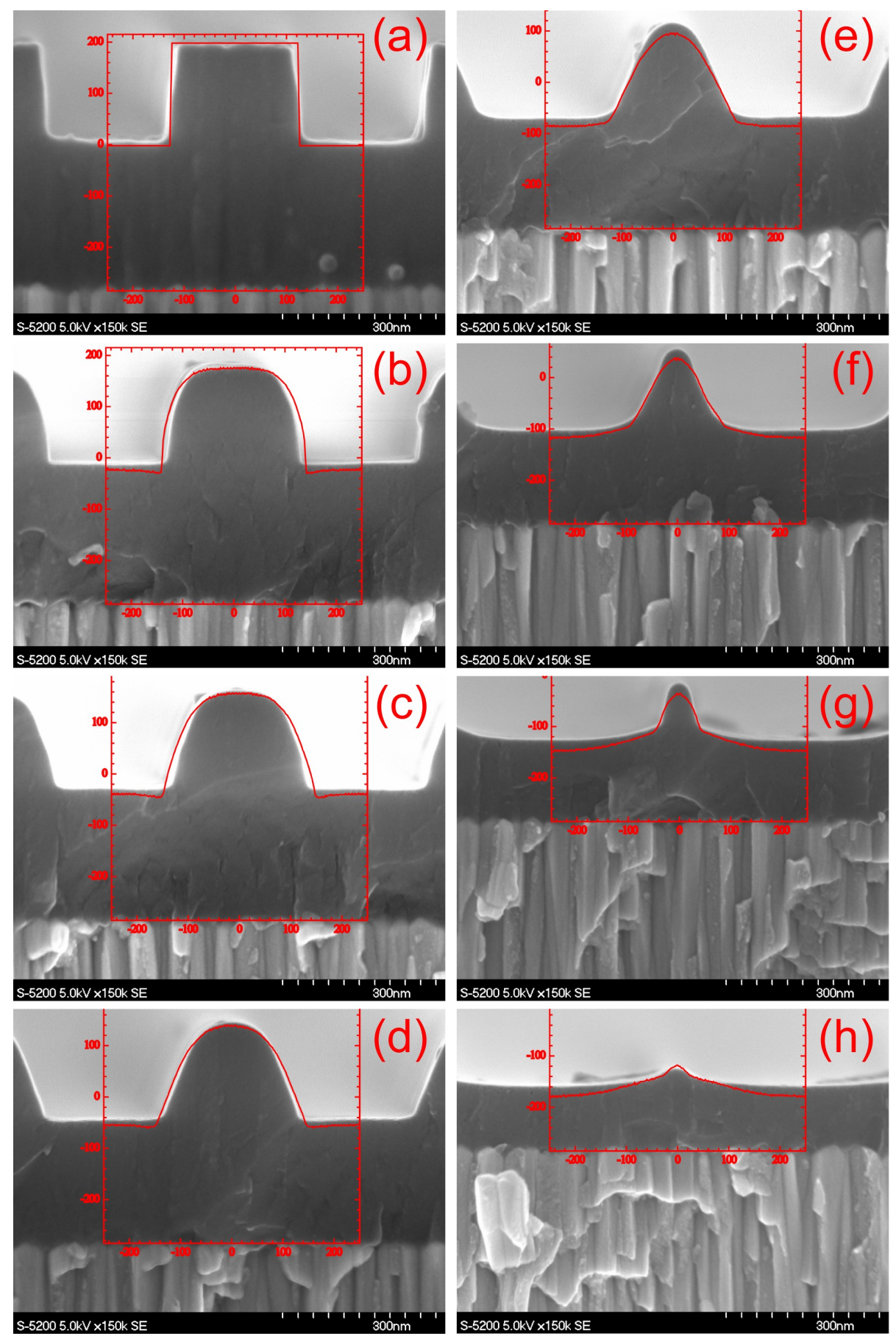

Figure 3. SEM images of a cross-section of the Si pitch grating following bombardment at the normal incidence. Red colored graphs are the surface profiles simulated by SDTrimSP-2D code. All scales are given in nanometers. Different images correspond to different incident fluence. The fluence value has been taken from the results of the simulation: (a) - initial surface state; (b) $-10 \times 10^{20} \mathrm{~m}^{-2}$; (c) $-15 \times 10^{20} \mathrm{~m}^{-2}$; (d) $-20 \times 10^{20} \mathrm{~m}^{-2}$; (e) $-30 \times 10^{20} \mathrm{~m}^{-2}$; (f) $40 \times 10^{20} \mathrm{~m}^{-2} ;(\mathrm{g})-50 \times 10^{20} \mathrm{~m}^{-2} ;(\mathrm{h})-60 \times 10^{20} \mathrm{~m}^{-2}$. 

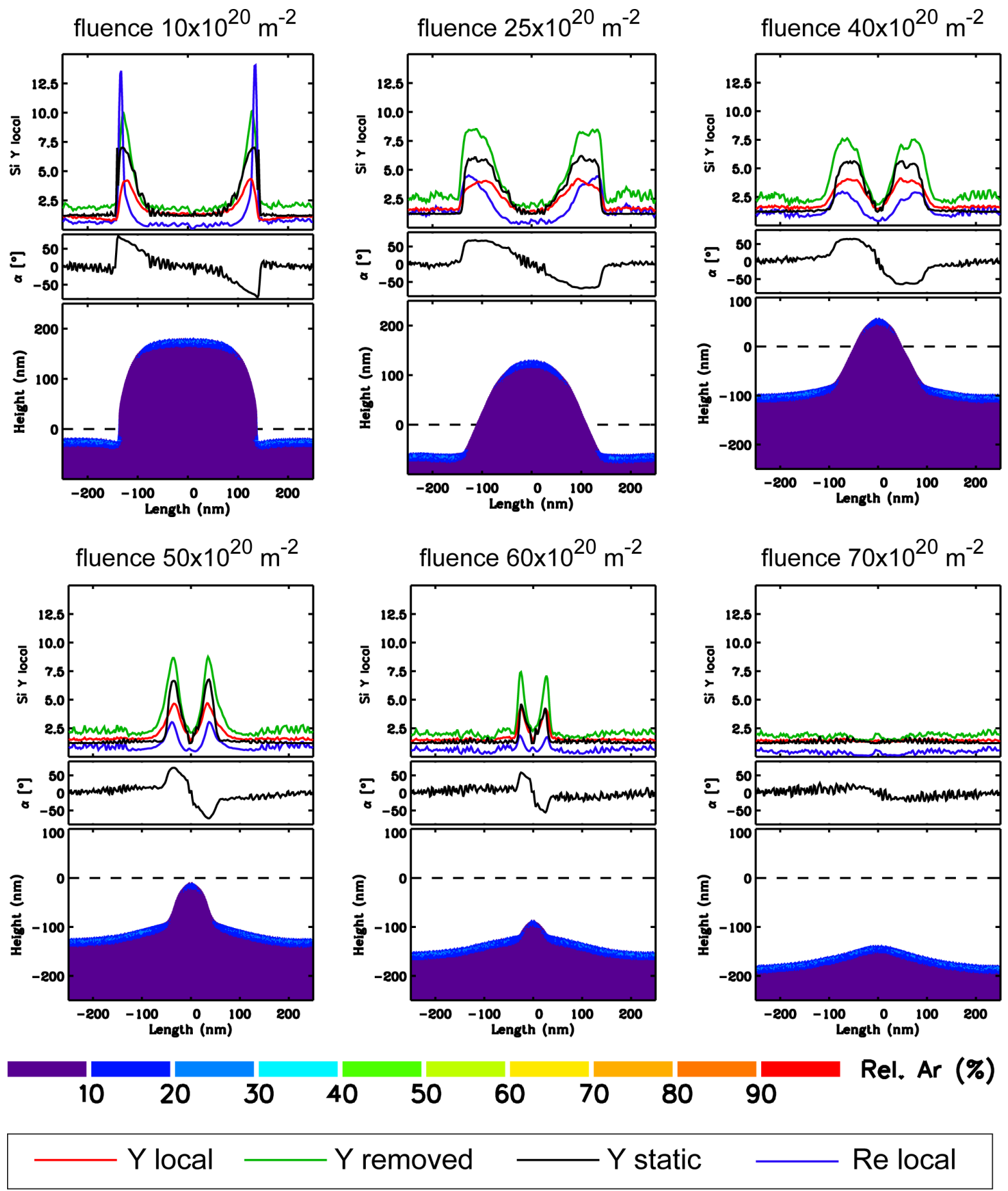

Figure 4. Calculated surface profiles; $Y_{\text {local }}$ (red lines), $Y_{\text {removed }}$ (green lines), $Y_{\text {static }}$ (black lines) and redeposition (blue lines) yields for different fluence values; the middle graph is the local angle of incidence. Colored regions on the profile correspond to the local Ar elemental concentration. All scales are given in nanometers. 

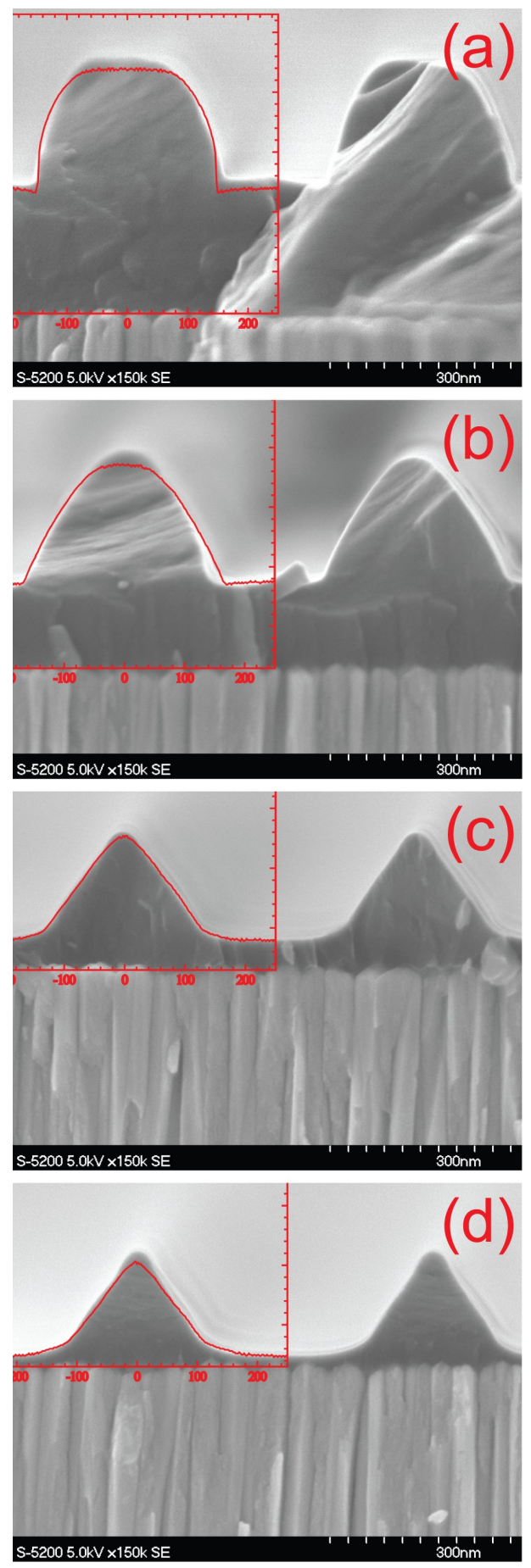

Figure 5. SEM images of a cross-section of the Si pitch grating following bombardment at an angle of $42^{\circ}$ parallel to the structure. Red colored graphs are the surface profiles simulated by SDTrimSP-2D code. All scales are given in nanometers. Different images correspond to different incident fluence. The fluence value has been taken from the results of the simulation: (a) $-10 \times 10^{20} \mathrm{~m}^{-2}$; (b) $-20 \times 10^{20} \mathrm{~m}^{-2}$; (c) $-35 \times 10^{20} \mathrm{~m}^{-2}$; (d) $-40 \times 10^{20} \mathrm{~m}^{-2}$. 


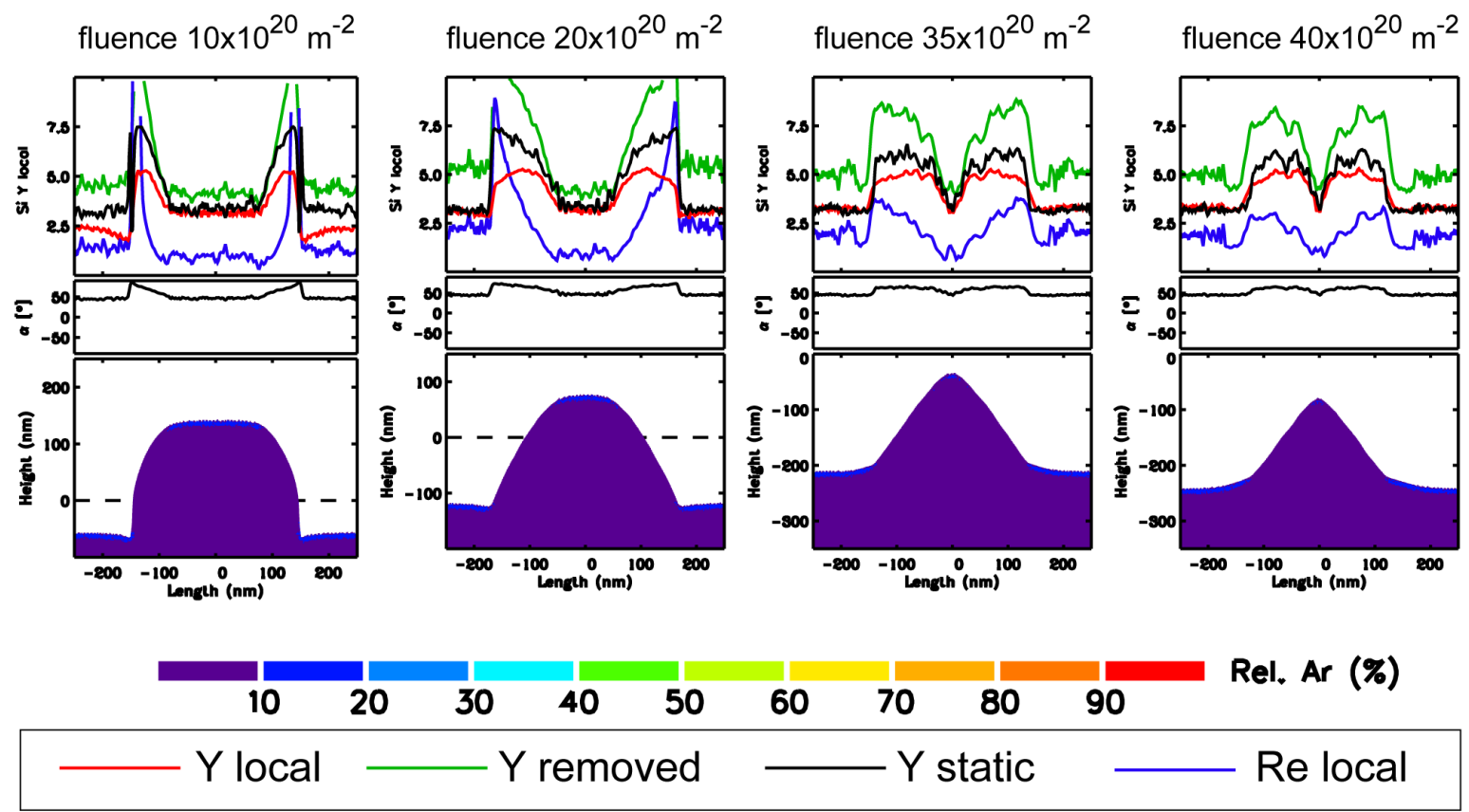

Figure 6. Calculated surface ; $Y_{\text {local }}$ (red lines), $Y_{\text {removed }}$ (green lines), $Y_{\text {static }}$ (black lines) and redeposition (blue lines) yields for different fluence values; the middle graph is the local angle of incidence. Colored regions on the profile correspond to the local Ar elemental concentration. All scales are given in nanometers. 

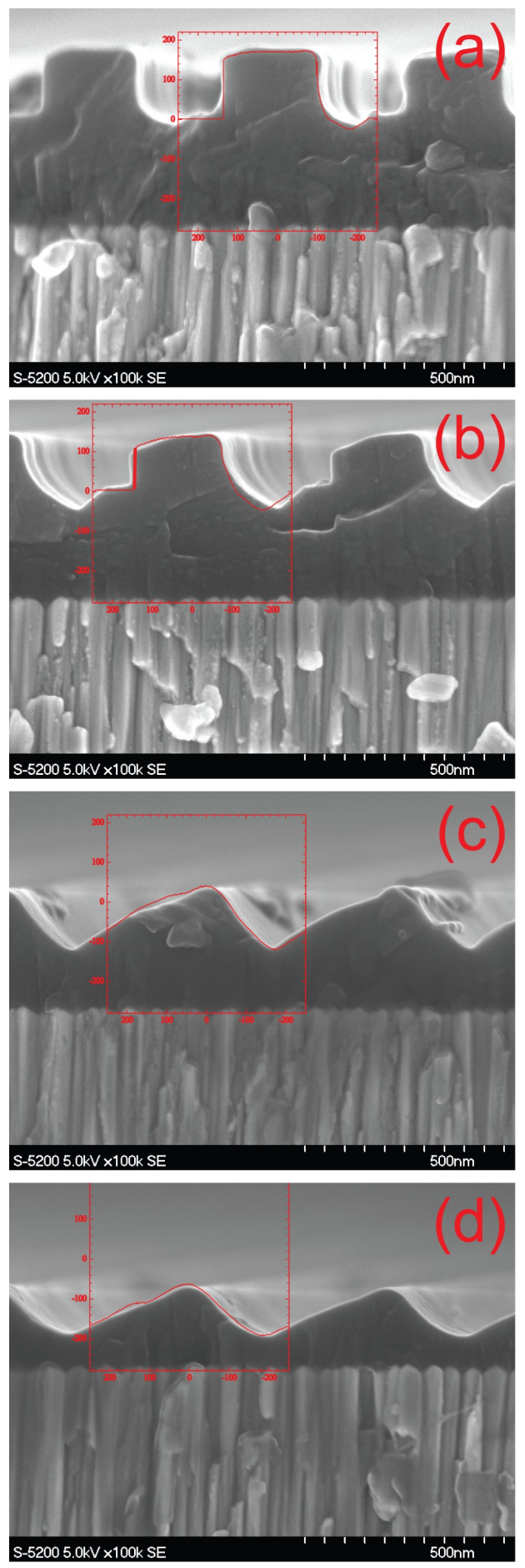

Figure 7. SEM images of a cross-section of the Si pitch grating following bombardment at an angle of $42^{\circ}$ perpendicular to the structure. Red colored graphs are the surface profiles simulated by SDTrimSP-2D code. All scales are given in nanometers. Different images correspond to different incident fluence. The fluence value has been taken from the results of the simulation: (a) $-5 \times 10^{20} \mathrm{~m}^{-2}$; (b) $-10 \times 10^{20} \mathrm{~m}^{-2}$; (c) $-25 \times 10^{20} \mathrm{~m}^{-2}$; (d) $-40 \times 10^{20} \mathrm{~m}^{-2}$. 


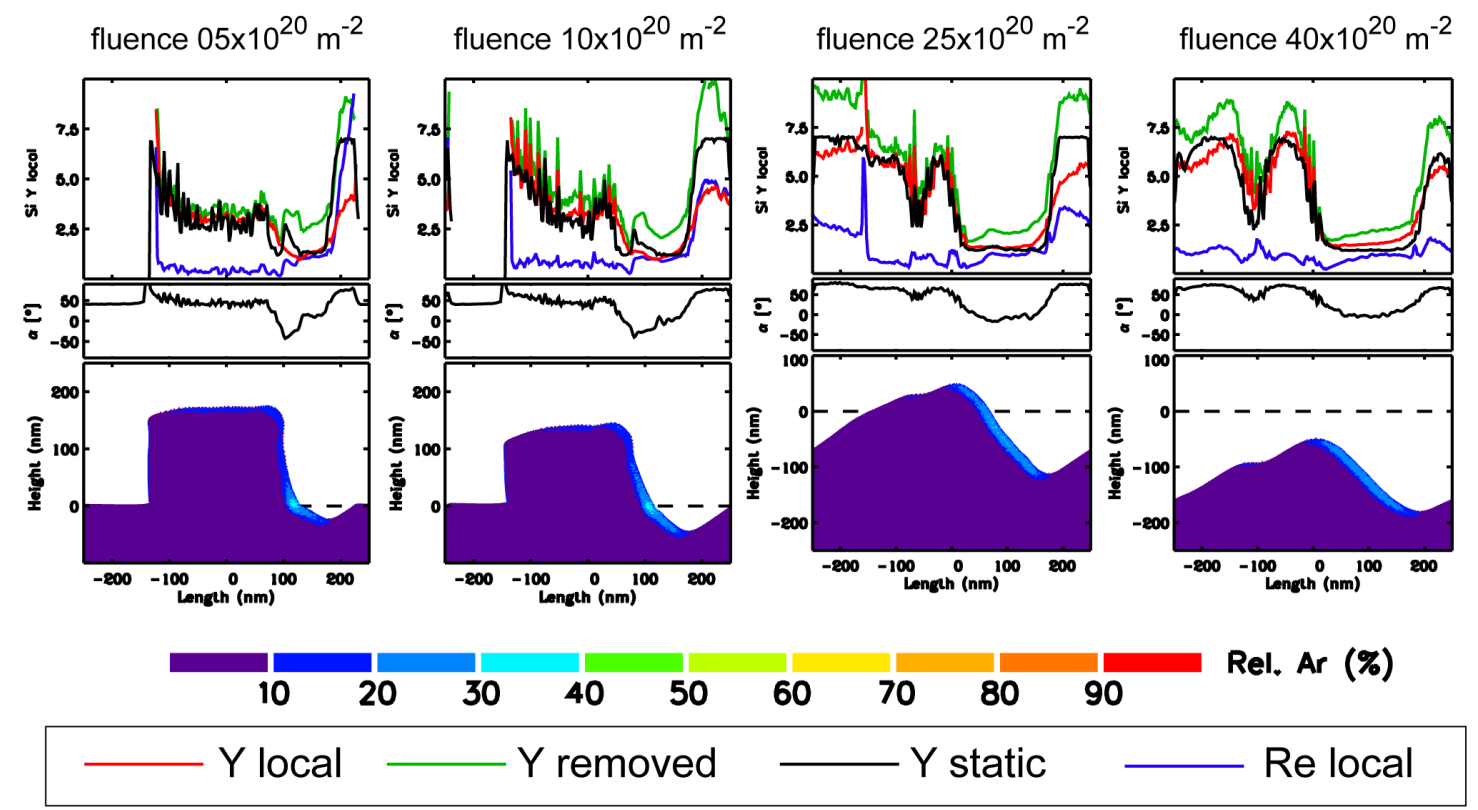

Figure 8. Calculated surface profiles; $Y_{\text {local }}$ (red lines), $Y_{\text {removed }}$ (green lines), $Y_{\text {static }}$ (black lines) and redeposition (blue lines) yields for different fluence values; the middle graph is the local angle of incidence. Colored regions on the profile correspond to the local Ar elemental concentration. All scales are given in nanometers. 